\title{
CATALOGUES OF INTERMEDIATE STARS \\ IN THE VICINITY OF RADIO SOURCES
}

\author{
I. KUMKOVA AND A. KOLOMIETS
}

Institute of Applied Astronomy, Russian Academy of Sciences

St. Petersburg, Russia

\begin{abstract}
The accurate positions of extragalactic radio sources are in use to link the radio and fundamental reference frames and contribute to determining relative orientation of HIPPARCOS and the radio reference frames as well as HIPPARCOS and FK5. Catalogues of intermediate stars chosen in the vicinity of extragalactic radio sources are an important part of the link and can be used for extension and densification of the HIPPARCOS frame. The description of main catalogues of intermediate stars is given. Results of investigation and comparison beetween CAMC, RRS2, PIRS and CRIS are presented.
\end{abstract}

\section{Introduction}

One of the most important tasks of modern astrometry is the extension of the radio coordinate frame - ICRF (International Celestial Reference Frame) adopted as new IAU reference frame (IAU Bull., 1995) to the optical catalogues such as Hipparcos and FK5. Up to now, only the fundamental optical system FK4/5 was accepted. At present, a new epoch for the Hipparcos catalogue is coming. That means that the Hipparcos catalogue can be recognized as optical counterpart of the ICRF due to complete sky coverage and accuracy of 1.5 mas which is essentially better than all previous optical catalogues. Recently, the frame of the Hipparcos catalogue has been fixed to that of the ICRF at the epoch 1991.25 with the accuracy of 0.6 mas. The residual rotation with time of the Hipparcos frame with respect to the ICRF is estimated to be of about 0.25 mas/year. The methods to link the different celestial reference frames such as radio, Hipparcos and fundamental ones, were developed successfully during the last decades.

I. M. Wytrzyszczak, J. H. Lieske and R. A. Feldman (eds.), Dynamics and Astrometry of Natural and Artificial Celestial Bodies, 505, 1997.

(C) 1997 Kluwer Academic Publishers. Printed in the Netherlands. 


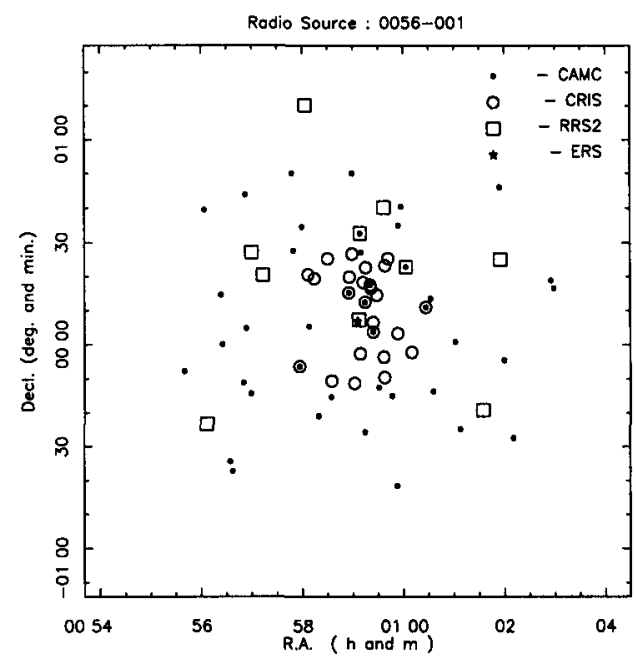

Figure 1. Distribution of stars from catalogues RRS2, CRIS, CAMC in area IAU 0056-001.

\section{Catalogues}

Up to now, the most fruitful way of establishing the link between different reference frames is the use of accurate positions of radio stars or extragalactic radio sources (ERS) obtained in both systems. These accurate positions, used to link radio and fundamental reference frames, contribute to the determination of the relative orientation of Hipparcos with respect to radio reference frames as well as to the FK5 frame. The optical positions of radio source counterparts are obtained by means of photographic astrometry. The multistep method of star position determination should be applied because of the significant stellar magnitude differences between the ERS and the catalogue stars. In this method two systems of intermediate stars in the vicinities of ERS are used: the first system of stars of $6^{m}-9^{m}$, whose positions are given in reference catalogues (PPM, Hipparcos), and the second system based upon stars of $11^{m}-14^{m}$, whose positions should be obtained in the process of this work. The selection of intermediate stars in both systems has a special importance as the positional accuracy and configuration of the stars influence the final radio source positions. Catalogues of intermediate stars chosen in ERS vicinities represent a very important part of the link between radio and optical reference frames. In the framework of different programs aiming to establish a link between radio and optical reference frames, different catalogues of intermediate reference stars are used. Among them, the meridian catalogues: RGO (Carlsberg), Bordeaux, Nikolaev, Leoncito; the photographic or CCD method: Hamburg, La Palma, 
USNO (southern station), Yale, Flagstaff; also the Bonn and Heidelberg Guide Stars catalogue; SLOAN: USNO.

At present, several catalogues of intermediate stars in radio sources vicinities have been issued. For all the catalogues the number of areas is of about 250 with centres mainly chosen from the same catalogue of ERS (Argue et al., 1984). The number of stars in any area can reach 30-40 stars. Different authors use their own criteria of selection of intermediate stars. This is why the area with the same centre and the same size can differ from one another. This is demonstrated in Figure 1 (area for ERS IAU 0056001). These catalogues have the following special features: first of all, they are very homogeneous in the sense of stellar magnitude, then they have a particular distribution on the celestial sphere, namely as clusters in selected areas. In general, they show a rather representative amount of stars covering the whole celestial sphere and can be used for astrometrical research. In this paper, we present the results of comparison and investigation of available catalogues of intermediate reference stars. The description of the catalogues is given in Table 1.

TABLE 1. Description of the catalogues.

\begin{tabular}{lccc}
\hline Cat. & $\begin{array}{c}\text { Number } \\
\text { of areas }\end{array}$ & $\begin{array}{c}\text { Number } \\
\text { of stars } \\
\text { in the area }\end{array}$ & $\begin{array}{c}\text { Radius } \\
\text { of areas } \\
\text { [arcmin] }\end{array}$ \\
\hline \multicolumn{4}{c}{ The First Reference System } \\
RRS2 & 232 & $9-12$ & $60-200$ \\
\multicolumn{4}{c}{ The Second Reference System } \\
CRIS & 232 & $25-31$ & $15-80$ \\
CAMC & 206 & $27-46$ & $20-190$ \\
\hline
\end{tabular}

The catalogues CRIS and PIRS (Kumkova et al., 1995) have been compiled in the framework of the CONFOR program (Cubanov et al. 1990, Kumkova et al. 1995). We investigated the CRIS as well as the PIRS but the versions are very close so that the presented results are similar for both catalogues. The epoch of the catalogues is rather close.

\section{Comparison and Calculations}

For comparison we took 232 common areas. At first, all these areas have been checked for homogeneous distribution. In Figure 2 the results of distribution analysis are presented for the selected area (for IAU 2201+315).

The data were obtained using of the Method of Dependences - Method of Schlesinger. Conditional weights or dependences have been calculated for 

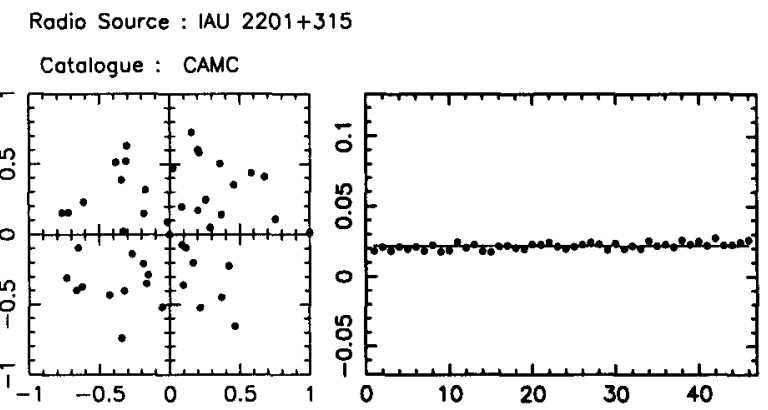

Radio Source : IAU $2201+315$

Catologue : CRIS
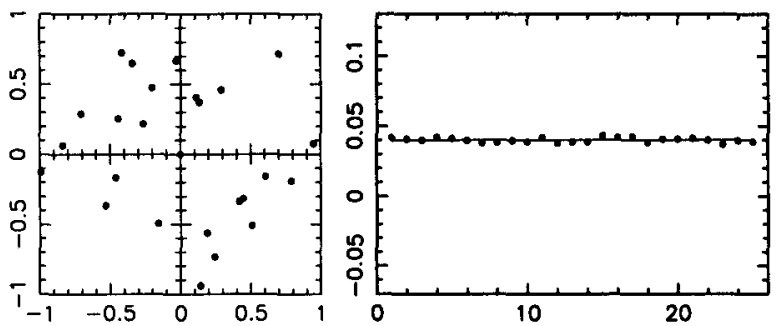

Figure 2. Distribution of stars in area IAU 2201+315 and estimation of dependences for catalogues CAMC and CRIS.

TABLE 2. Results of the catalogue investigation.

\begin{tabular}{lccrrr}
\hline Cat. & $\begin{array}{c}\text { Number of } \\
\text { homogeneous } \\
\text { areas }\end{array}$ & $\begin{array}{c}\text { Number of } \\
\text { unhomogeneous } \\
\text { areas }\end{array}$ & $\begin{array}{c}\text { Number } \\
\text { of areas } \\
40^{\prime}-80^{\prime}\end{array}$ & $>80^{\prime}$ \\
\hline CRIS & 178 & 54 & 216 & 12 & 4 \\
CAMC & 64 & 142 & 87 & 107 & 12 \\
\hline
\end{tabular}

every star from the area according to standard procedure (Deutch, 1973). In the case of homogeneous distribution of stars, the values of weights are equal to $1 / \mathrm{N}$, where $\mathrm{N}$ is the number of stars in the area. The deviation from homogeneous distribution is manifested by the difference between the obtained values of weights and $1 / \mathrm{N}$. The results of such an investigation for CRIS and SRS CAMC (CAMC N 4, 1989) are shown in Table 2.

The RRS2 (Tel'nyuk-Adamchuk and Molotaj, 1989) catalogue contains the reference stars of the first intermediate system but its number is not sufficient enough to provide the position of ERS in the Hipparcos frame with the necessary accuracy. At present, a new catalogue (NC) is being 
compiled including intermediate reference stars of the first reference system. The positions of the stars in NC are given as in the Hipparcos as well as in the PPM catalogue. The distribution of the RRS2 and NC stars in the area centred on IAU $0735+178$ is presented in Figure 3. Results of positional determination for the stars are presented in Table 3.

TABLE 3. Positions of faint stars in Hipparcos frame around the IAU $1252+119$.

\begin{tabular}{cccccc}
\hline HSTGC & $\alpha$ & $\sigma_{\alpha}$ & \multicolumn{1}{c}{$\delta$} & $\sigma_{\delta}$ \\
\hline 501194 & $12^{h} 55^{m} 53^{s} \cdot 161$ & $0^{s} .008$ & $11^{\circ} 30^{\prime} 32^{\prime \prime} 90$ & $0^{\prime \prime} 12$ \\
501131 & 125600.241 & 0.010 & 112812.32 & 0.15 \\
501169 & 125523.906 & 0.010 & 113350.33 & 0.15 \\
501011 & 125506.917 & 0.008 & 112240.84 & 0.14 \\
501008 & 125440.005 & 0.010 & 112231.91 & 0.15 \\
501185 & 125412.796 & 0.008 & 113013.06 & 0.12 \\
501186 & 125400.034 & 0.010 & 113006.92 & 0.15 \\
501193 & 125322.774 & 0.009 & 113039.39 & 0.13 \\
500848 & 125329.751 & 0.010 & 115823.73 & 0.15 \\
500809 & 125353.445 & 0.009 & 115752.94 & 0.13 \\
500765 & 125358.078 & 0.008 & 115123.35 & 0.12 \\
501179 & 125515.541 & 0.009 & 114026.34 & 0.14 \\
500753 & 125537.347 & 0.010 & 115040.52 & 0.15 \\
500733 & 125528.743 & 0.010 & 115602.90 & 0.15 \\
501096 & 125528.028 & 0.008 & 115932.17 & 0.12 \\
500743 & 125602.078 & 0.008 & 115458.69 & 0.12 \\
500778 & 125546.707 & 0.010 & 115131.58 & 0.15 \\
500767 & 125526.467 & 0.008 & 115318.69 & 0.12 \\
\hline
\end{tabular}

Moreover, this catalogue of intermediate stars of the first system gives the opportunity to study local systematic differences between these catalogues and to estimate the local systematic errors of PPM at the adopted epoch.

\section{Conclusions}

Thus, the catalogues of intermediate stars of both steps can provide:

- excellent data for studies of reference systems;

- maintenance of the Hipparcos frame based upon periodical redetermination of it's rotation parameters;

- extension of the Hipparcos catalogue to fainter stars. 


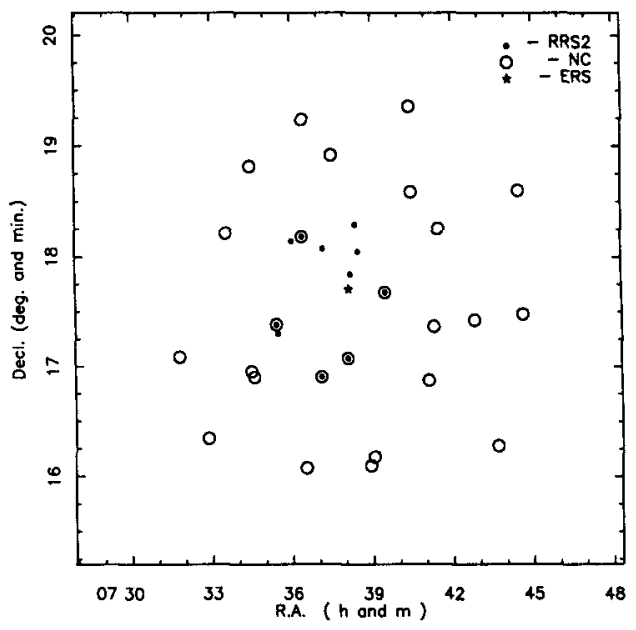

Figure 3. Distribution of stars from catalogues RRS2 and NC in area IAU $0735+178$.

This determined the necessity of reducing the catalogues of intermediate stars to the one system and actuality of compiling the the catalogue to fulfillment of the tasks mentioned above.

\section{References}

IAU Resolution B6: 1995, IAU Bull. 74, 01-23.

Kumkova, I.I., Kolomietz, A.M., Solina, N.I., and Tel'nyuk-Adamchuk, V.V.: 1995, "The catalogue of stars of 12-14 stellar magnitude in radio source vicinities", Tech. Note of VINITI 1571-B95, 01-122 (in Russian).2

Gubanov, V.S., Kumkova, I.I., and Tel'nyuk-Adamchuk, V.V.: 1990, "CONFOR: A new program for the determining connection between radio and optical reference frames", in: Inertial Coordinate System on the Sky, IAU Symposium 141 (J.H. Lieske, V.K. Abalakin, eds), Kluwer, Dordrecht, 75-76.

Kumkova, I.I., Tel'nyuk-Adamchuk, V.V., Babenko, Yu.G., and Vertopolokh, O.Ya.: 1995, "CONFOR program: Determination of relative orientation paremeters between VLBI and FK5 reference frames", in: Astronomical and Astrophysical Objectives of Sub-Milliarcsecond Optical Astrometry, IAU Symposium 166 (E. Høg, P.K. Seidelmann, eds), 1994.

Argue, A.N., de Vegt, Ch., Elsmore, R., Fanselow, J., Harrington, P., Hemenway, P., Johnston, K.J., Kühr, H., Kumkova, I., Niell, A.E., Walter, H.G., and Witzel, A.: 1984, "A catalog of selected compact radio sources for the construction of an extragalactic radio/optical reference frame", Astron. Astrophys. 130, 191-199.

Carlsberg Meridian Catalogue $N$ 4: 1989, Copenhagen Univ. Obs., R. Greenwich Obs., Inst. y Obs. de San Fernando, eds, La Palma.

Tel'nyuk-Adamchuk, V.V. and Molotaj, A.A.: 1989, "The catalogue of the intermediate stars of 6-9 stellar magnitude in vicinities of extragalactic radio sources", Tech. Note of Ukrainian Institute of Scientific and Technical Information 1459-Uk89, 01-111.

Deutch, A.N.: 1973, Course of Astrophysics and Stellar Astronomy, 1, Nauka, Moskva, 229-232 (in Russian). 